\title{
Cognition Cloud Model for Next Generation Mobile Internet: Communication, Control, and Application
}

\author{
Yong Jin, ${ }^{1}$ Chaobo Yan, ${ }^{2}$ and James Nightingale ${ }^{3}$ \\ ${ }^{1}$ Changshu Institute of Technology, Changshu 215500, China \\ ${ }^{2}$ University of Michigan, Ann Arbor, MI, USA \\ ${ }^{3}$ University of the West of Scotland, Paisley PA1 2BE, UK \\ Correspondence should be addressed to Yong Jin; jinyong@cslg.cn \\ Received 7 March 2016; Accepted 17 March 2016 \\ Copyright (@) 2016 Yong Jin et al. This is an open access article distributed under the Creative Commons Attribution License, which \\ permits unrestricted use, distribution, and reproduction in any medium, provided the original work is properly cited.
}

It is well known that the next generation of mobile Internet has arrived, bringing with it great technical challenges and immense opportunities to enhance both our personal lives and work environments. A new mobile Internet has been the proliferation of a large and increasingly diverse set of mobile services and applications. To accommodate this rapid development of next generation mobile Internet services and applications, new reliable, trusted, and high performance cognition cloud model-based services for communication, control, and mobile computational services are required.

This special issue consists of six papers. Three of the papers focus on cloud computing: a paper on cooperative cloud service aware mobile Internet coverage connectivity guarantee protocol, a paper on cooperative optimization QoS cloud routing protocol, and a paper on user utility oriented queuing model. Another two papers are focused on data mining: one paper discusses the regression cloud models of data center and the other examines the mobile Internet service opportunistic drive and application aware data mining. The remaining paper studies the cooperative cognitive cloud and opportunistic weight particle swarm.

In the paper "Cooperative Cloud Service Aware Mobile Internet Coverage Connectivity Guarantee Protocol Based on Sensor Opportunistic Coverage Mechanism," Q. Qin et al. proposed the coverage connectivity guarantee protocol for mobile Internet for improving the Internet coverage ratio and providing connectivity guarantee, based on sensor opportunistic coverage mechanism and cooperative cloud service.
In the paper "Cooperative Optimization QoS Cloud Routing Protocol Based on Bacterial Opportunistic Foraging and Chemotaxis Perception for Mobile Internet," S. Wang et al. established the scheme of optimizing the cloud routing efficiency based on opportunistic bacterial foraging bionics for strengthening the mobile Internet mobility management and cloud platform resources utilization. They also put forward a chemotaxis perception of collaborative optimization QoS (Quality of Services) cloud routing mechanism.

In the paper "User Utility Oriented Queuing Model for Resource Allocation in Cloud Environment," Z. Zhang and Y. Li proposed a user utility oriented queuing model for task scheduling. They modeled task scheduling in cloud environment as an $\mathrm{M} / \mathrm{M} / 1$ queuing system. They classified the utility into time utility and cost utility and built a linear programming model to maximize total utility for both of them.

In the paper "Regression Cloud Models and Their Applications in Energy Consumption of Data Center," Y. Zhou et al. analyzed linear and nonlinear regression energy model based on performance counters and system utilization and proposed a support vector regression energy model. They gave a general linear regression framework and compared three linear regression models.

In the paper "Cloud Platform Based on Mobile Internet Service Opportunistic Drive and Application Aware Data Mining," G. Zhou presented a reliable and efficient data mining cloud platform construction scheme based on the mobile Internet service opportunistic driving and application 
perception. Their scheme can satisfy the diversity of mobile Internet service and deal with the inefficient data mining problems.

In the paper "QoE Guarantee Scheme Based on Cooperative Cognitive Cloud and Opportunistic Weight Particle Swarm," W. Shi proposed the QoE (Quality of Experience) guarantee mechanism based on construction algorithms of cooperative cognitive cloud platform and optimization algorithm of opportunities weight particle swarm clustering.

It is evident that this special issue does not cover many other important areas of relevance to cloud model for next generation mobile Internet. Nonetheless, we hope that readers will find the information presented to be interesting and useful.

\section{Acknowledgments}

We thank all the authors who responded to the call for papers. We also wish to acknowledge all the reviewers for their dedicated efforts in ensuring a high standard for the selected papers.

Yong Jin

Chaobo Yan

James Nightingale 


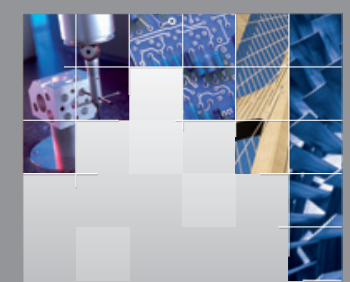

\section{Enfincering}
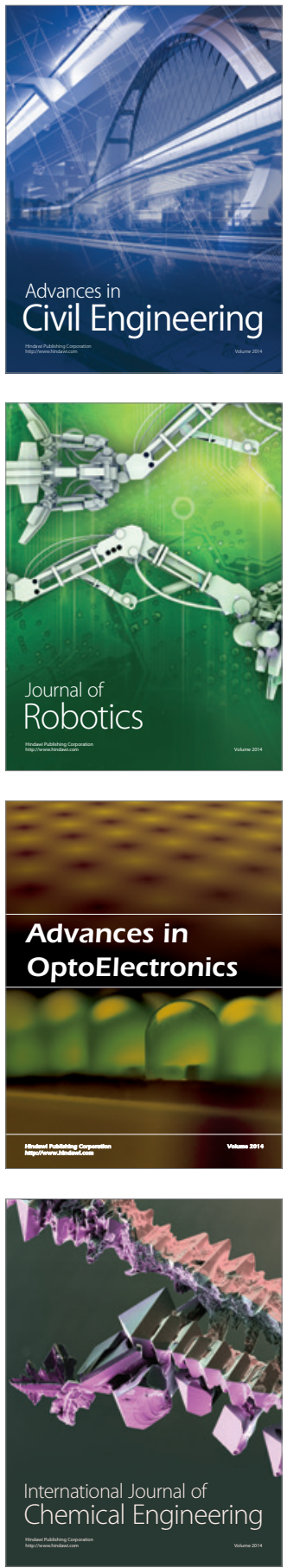

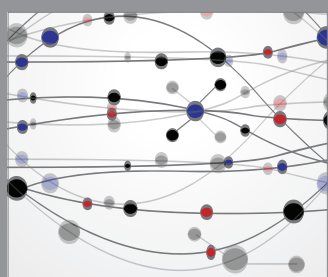

The Scientific World Journal

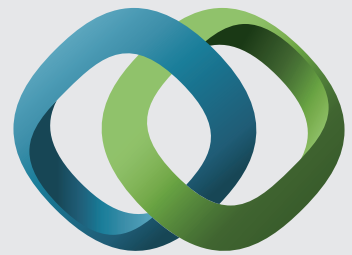

\section{Hindawi}

Submit your manuscripts at

http://www.hindawi.com
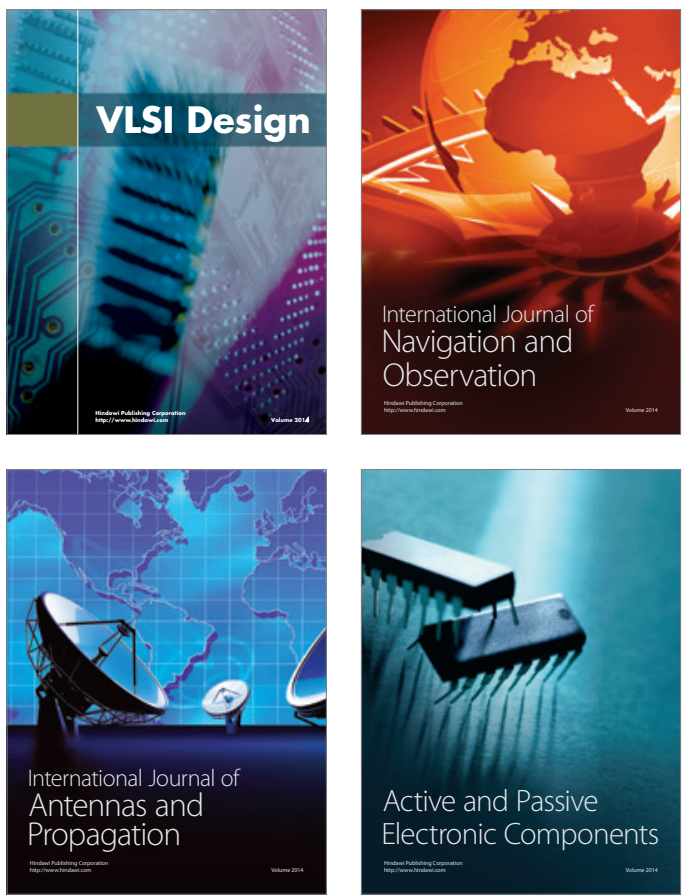
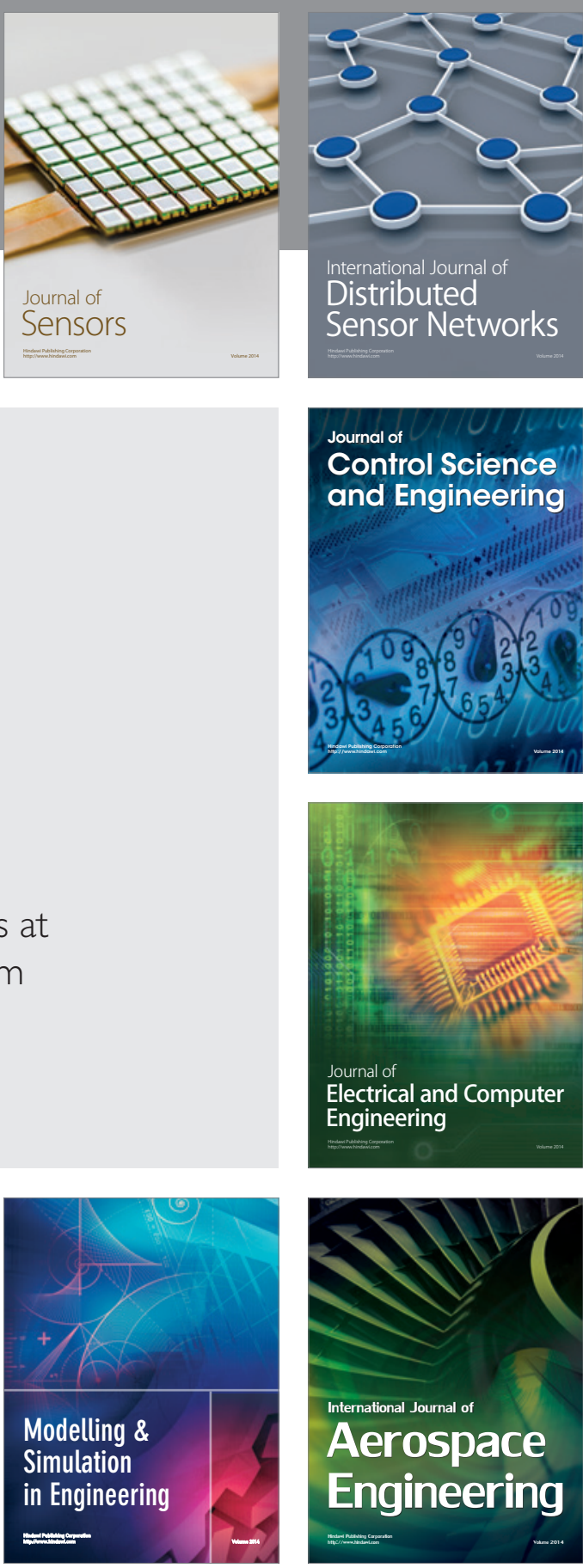

International Journal of

Distributed

Sensor Networks

Journal of

Control Science

and Engineering
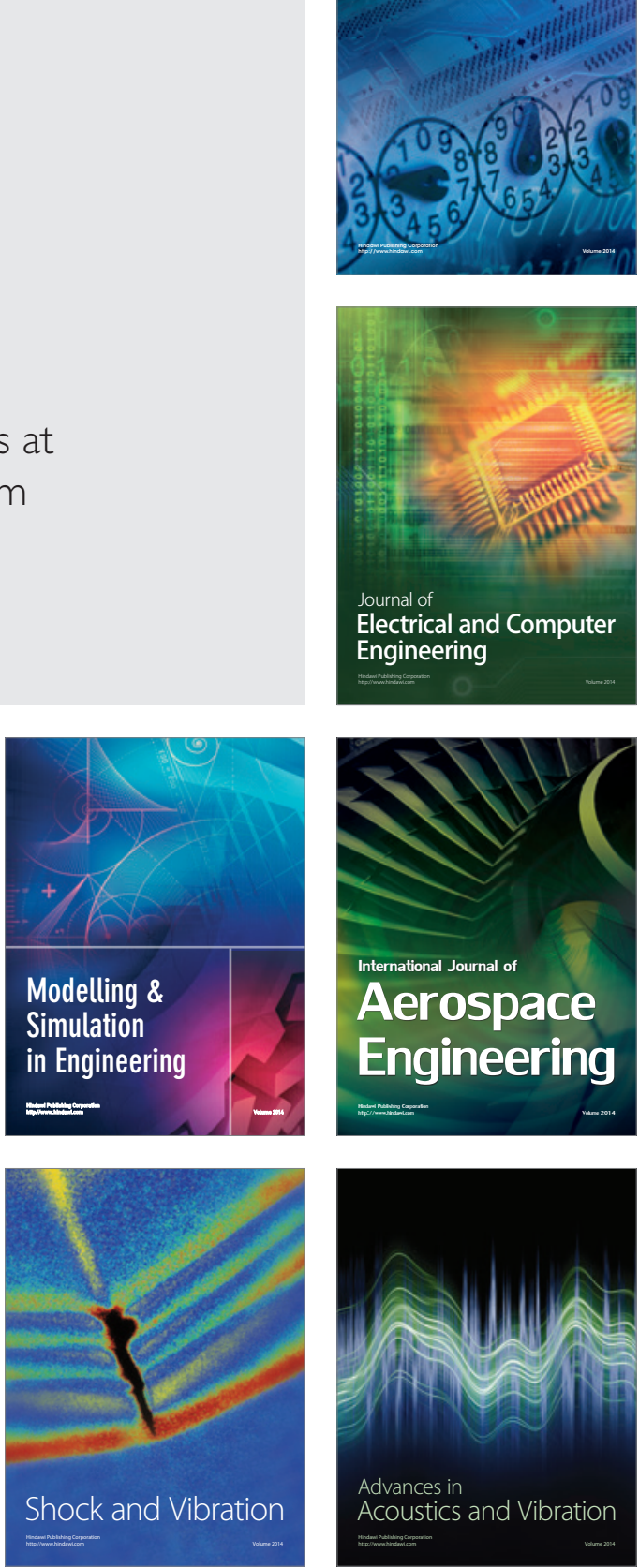